\title{
Cuidados paliativos no câncer e os princípios doutrinários do SUS
}

\author{
Palliative cares on cancer and the doctrinal principles of SUS
}

Ernani Costa Mendes' ${ }^{\mathbf{1}}$ Luiz Carlos Fadel de Vasconcellos ${ }^{\mathbf{2}}$

RESUMO Os cuidados paliativos são destinados às pessoas com doenças crônicas evolutivas que ameaçam suas vidas. Têm como característica preservar a qualidade da vida, do diagnóstico ao estágio final, e guardam coerência com a doutrina do Sistema Único de Saúde (SUS): universalidade, integralidade, equidade. Este artigo, focado no câncer, faz uma reflexão sobre a cobertura da população, sua organização na atenção básica, média e alta complexidades e formação de profissionais, a partir de revisão bibliográfica e dos instrumentos normativos. Conclui-se que a estruturação da rede de cuidados paliativos carece de eficácia para o seu desenvolvimento, enquanto política pública coerente com a doutrina do SUS.

PALAVRAS-CHAVE Câncer; Cuidados paliativos; Sistema Único de Saúde.

ABSTRACT Palliative cares are allocated to people with evolutionary chronic diseases which threaten their lives. They are characterized for preserving the quality of life from diagnosis to late stage, and keep consistency with the doctrine of the Unified Health System (SUS): universality, integrality, equity. This article, focused on cancer, reflects on the coverage of the population, their organization in basic, medium and high complexities and training of professionals, from literature review and regulatory instruments. It is concluded that the structuring of palliative care network lacks effectiveness for its development, while consistent public policy with the doctrine of SUS.

KEYWORDS Cancer; Palliative care; Unified Health System.

1Fundação Oswaldo Cruz (Fiocruz), Escola Nacional de Saúde Pública Sérgio Arouca (Ensp) - Rio de Janeiro, Brasil.

ernani-mendes@ig.com.br

2 Fundação Oswaldo Cruz (Fiocruz), Escola Nacional de Saúde Pública Sérgio Arouca (Ensp) - Rio de Janeiro, Brasil. elfadel@globo.com 


\section{Introdução}

O número de casos de câncer vem crescendo anualmente, representando a segunda causa de morte no Brasil e no mundo. "Responde por 20\% dos óbitos na Europa, com mais de três milhões de novos casos e 1,7 milhões de óbitos por ano" (INCA, 2012, P. 15). O câncer constitui-se numa importante questão de saúde pública, tanto nos países desenvolvidos quanto nos países em desenvolvimento, em virtude de seu crescente impacto sobre a rede de serviços e sobre a agenda de ações que atendam à exigibilidade da atenção. Diante de estimativas de redução da ocorrência de câncer em até $30 \%$ dos casos, por meio de medidas preventivas, evidencia-se a necessidade de políticas sanitárias mais abrangentes e robustas (FIOCRUZ, 2012). Inclusive, em algumas situações, o câncer pode ser totalmente prevenível, caso do mesotelioma de origem ocupacional, conforme assinala o documento 'Diretrizes para a vigilância do câncer relacionado ao trabalho' (INCA, 2012).

Chama a atenção a desigualdade dessa situação, uma vez que mais de $70 \%$ de todas as mortes por câncer ocorrem nos países em vias de desenvolvimento. A carga do câncer continuará aumentando nos países em desenvolvimento e crescerá ainda mais em países desenvolvidos se medidas preventivas não forem amplamente aplicadas. Nestes, os tipos de câncer mais frequentes na população masculina são os de próstata, pulmão, cólon e reto; e mama, cólon e reto e pulmão entre as mulheres. Nos países em desenvolvimento, os três cânceres mais frequentes em homens são pulmão, estômago e fígado; e mama, colo do útero e pulmão nas mulheres (INCA, 2014).

Segundo estimativas de incidência de câncer do projeto Globocan 2012, da Agência Internacional para Pesquisa em Câncer (IARC - International Agency for Research on Cancer), da Organização Mundial da Saúde (OMS), houve 14,1 milhões de casos novos de câncer e um total de 8,2 milhões de mortes por câncer em todo o mundo, em 2012. A OMS estima, ainda, para o ano de 2030, 21,4 milhões de casos incidentes, 13,2 milhões de mortes e 75 milhões de pessoas vivas, anualmente, com câncer (INCA, 2014).

No Brasil, o Instituto Nacional de Câncer José de Alencar Gomes da Silva (Inca), que é o responsável pela política nacional integrada para o controle e a prevenção do câncer, estimou para o biênio de 2014/2015 a ocorrência de 576 mil casos novos. O câncer de pele do tipo não melanoma (182 mil casos novos) é o mais incidente na população brasileira, seguido pelos tumores da próstata (69 mil), mama feminina (57 mil), cólon e reto (33 mil), pulmão (27 mil), estômago (20 mil) e colo do útero (15 mil), acompanhando o mesmo perfil da magnitude observada para a América Latina (INCA, 2014).

Segundo o IBGE (2009), o aumento da expectativa de vida, o envelhecimento e a mudança do comportamento da densidade demográfica do Brasil projetam estimativas de que o grupo etário de 60 anos ou mais, duplicará em termos absolutos no período de 2000 a 2020 , ao passar de 13,9 para 28,3 milhões, elevando-se, em 2050, para 64 milhões.

O envelhecimento populacional constitui um dos maiores desafios para a saúde pública contemporânea, especialmente em países em desenvolvimento, onde esse fenômeno ocorre em ambientes de pobreza e grande desigualdade social. Em 2025, estima-se que, dos dez países do mundo com o maior número de idosos, cinco serão países em desenvolvimento, entre eles, o Brasil (LIMA-COSTA, 2003).

Em recente documento sobre a prospecção estratégica do sistema de saúde brasileiro para 2030, elaborado pela Fundação Oswaldo Cruz, em parceria com outras instituições brasileiras, é assinalado que envelhecimento e doenças crônicas, em determinado momento, para uma determinada população, acionam todos os níveis de assistência, "e nenhum deles poderia 
ser chamado de 'resolutivo' no sentido clássico associado à ideia de cura" (FIOCRUZ, 2012, P. 141). O fato requer a intervenção de distintas especialidades do campo da saúde em todos os níveis. Além disso, especialmente no nível primário, frequentemente, ultrapassam-se as fronteiras dos serviços de saúde, abrangendo as áreas sociais e de apoio comunitário. O mesmo documento ainda observa que as demais formas de cuidados em saúde, muitas delas utilizadas em países desenvolvidos, "como assistência domiciliar, centros de cuidados prolongados e de cuidados paliativos, crescem em importância [...]", e, apesar desse fato, “[...] sua utilização carece de avaliação e planejamento no Brasil” (FIOCRUZ, 2012 P. 141).

Atualmente, a doença crônica, progressiva e incurável é a principal causa de incapacidades funcionais, sofrimento e morte, como é o caso do câncer, da Aids e de outras enfermidades que acometem os diversos órgãos vitais. No caso específico do câncer, que tem seu crescimento progressivo em todas as faixas etárias e é descoberto em estadiamento tardio, no Brasil, com taxas de incidência crescentes e de incapacidades de toda ordem para os pacientes, há uma tendência a ocorrerem grandes demandas ao sistema de saúde (FIOCRUZ, 2012; HENNEMANN-KRAUSE, 2012; LIMACOSTA, 2003).

Segundo dados do IBGE (2009), as mais recentes informações da PNAD (Pesquisa Nacional por Amostra de Domicílios) que tratam do tema mostram que, do total da população residente brasileira, 31,3\% afirmaram ter pelo menos uma doença crônica, correspondendo a 59,5 milhões de pessoas. O percentual de mulheres com doenças crônicas $(35,2 \%)$ é maior do que o de homens (27,2\%), e aumenta com a idade: $45 \%$ para a população de 40 a 49 anos de idade e $79,1 \%$ na população de 65 anos ou mais de idade. A doença crônica mais frequentemente relatada foi a hipertensão arterial (14,1\%), seguida de doenças da coluna (13,5\%).

Recentemente, no Brasil, foi lançado o Plano de Ações Estratégicas para o
Enfrentamento das Doenças Crônicas não Transmissíveis (DCNT), para o decênio 2011-2022, que as aponta como um problema de saúde de grande magnitude. São responsáveis por $72 \%$ das causas de morte, com destaque para doenças do aparelho circulatório (31,3\%), câncer (16,3\%), diabetes $(5,2 \%)$ e doença respiratória crônica $(5,8 \%)$, e atingem indivíduos de todas as camadas socioeconômicas e, de forma mais intensa, aqueles pertencentes a grupos vulneráveis, como os idosos e os de baixas escolaridade e renda. O plano tem como objetivo principal ações que visam ao fortalecimento da capacidade de resposta do Sistema Único de Saúde (SUS) e à ampliação das ações de cuidado integral para a prevenção e o controle das DCNT (BRASIL, 2011).

Com relação à questão específica do câncer, a atenção oncológica no Brasil, de 2005 a 2013, foi norteada pela Portaria $n^{\circ}$ $2.439 / \mathrm{GM}$, de 08/12/2005, que instituiu a Política Nacional de Atenção Oncológica. Para dar-lhe cumprimento, nesse período, o SUS estruturou respostas para melhor organizar a atenção ao câncer, levando-se em conta as dimensões e as heterogeneidades cultural e socioeconômica do Brasil. Nesse sentido, o Ministério da Saúde publicou, além da Portaria citada, a de $n^{\circ} 741$, de 19/12/2005. Enquanto a primeira adotava os parâmetros da instituição da política: promoção, prevenção, diagnóstico, tratamento, reabilitação e cuidados paliativos, a ser implantada em todas as unidades federadas, respeitadas as competências das três esferas de gestão (BRASIL, 2005A), a segunda determinava novas classificações e requisitos para os estabelecimentos que tratam câncer (Centros e Unidades de Alta Complexidade em Oncologia), além de propor parâmetros para o planejamento e a avaliação da Rede de Alta Complexidade em Oncologia e de estabelecer procedimentos para melhoria das informações sobre a atenção ao câncer (Registro Hospitalar de Câncer - RHC) (BRASIL, 2005B). 
Coerente com a relevância da questão do câncer, na perspectiva da sua atenção integral foi instituída a Lei Presidencial $\mathrm{n}^{\mathrm{o}} 12.732$ (22/11/2012), que dispõe sobre o primeiro tratamento de paciente com neoplasia maligna comprovada e estabelece prazo para seu início. Para tentar diminuir o retardamento do diagnóstico do câncer, a lei estabelece prazo para o paciente ser tratado no SUS. Em seu Art. $2^{\circ}$, assegura que o paciente com neoplasia maligna tem direito ao primeiro tratamento no SUS no prazo de até 60 dias, contados a partir do dia em que for firmado o diagnóstico em laudo patológico ou em prazo menor, conforme a necessidade terapêutica do caso, registrada em prontuário único.

E, em 2013, considerando sua importância epidemiológica e sua magnitude como problema de saúde pública e a necessidade de redução da mortalidade e da incapacidade, foi editada a nova Portaria, que trata da política nacional de atenção ao câncer. Guardando coerência com o eixo III do mencionado Plano de Ações Estratégicas para o Enfrentamento das DCNT, a Portaria $n^{\circ}$ 874, de 16/05/2013, (BRASIL, 2013, P. 129) “institui a Política Nacional para a Prevenção e Controle do Câncer na Rede de Atenção à Saúde das Pessoas com Doenças Crônicas no âmbito do Sistema Único de Saúde (SUS)" (PNPCC-RAS), sendo da responsabilidade do Ministério da Saúde estabelecer diretrizes nacionais para a prevenção e o controle do câncer, estimulando a atenção integral e articulando as diversas ações nos três níveis de gestão do SUS. O objetivo central da política é contribuir para a melhoria da qualidade de vida dos usuários com câncer, por meio de ações de promoção, prevenção, detecção precoce, tratamento oportuno e cuidados paliativos.

Este texto tem como objetivo geral analisar a relação entre os cuidados paliativos e os princípios doutrinários do SUS. Partindo da questão 'se' e 'como' esses princípios são contemplados nas ações propostas de cuidados paliativos inseridos na política, a hipótese do texto é que a implementação e a organização dos cuidados paliativos carecem, ainda, de mecanismos mais eficazes para o seu desenvolvimento, dentro da perspectiva de uma política pública de saúde, coerente com a doutrina do sistema.

\section{Metodologia}

A metodologia utilizada foi a revisão bibliográfica, considerando duas vertentes de abordagem: uma com foco no estado da arte, utilizando descritores relacionados ao tema (Sistema Único de Saúde e cuidados paliativos) nas bases de dados Bireme, Scielo, PubMed e Capes, a partir de 2005 (ano da publicação da primeira portaria nacional sobre o tema), e a outra referente a documentos legais emitidos pelo nível nacional do SUS, tais como portarias, decretos e leis, também a partir de 2005, coletados nos sites oficiais do governo federal.

\section{Cuidados paliativos no câncer e os princípios do SUS}

De acordo com a Política Nacional de Prevenção e Controle do Câncer, estabelecida na Portaria $n^{0} 874 / 2013$, os cuidados paliativos estão inseridos em todos os níveis de atenção na área de saúde, respeitando o conceito de hierarquização da assistência no âmbito do SUS, que se traduz na atenção básica de saúde, na média e na alta complexidades, garantindo, com isso, o direito integral, equânime e universal à saúde do cidadão. O ponto nodal dos cuidados paliativos, no âmbito da Política, depende de uma compreensão mais elaborada do seu significado e de suas dimensões. Coerente com a estratégia de enfrentamento das doenças crônicas não transmissíveis, a perspectiva 
é de ampliar, fortalecer e qualificar a assistência oncológica no SUS, capacitando, para isso, servidores da rede para cuidados paliativos no decênio 2011-2022, tendo em vista a maior e sempre crescente demanda do número de pessoas que precisam e precisarão de cuidados paliativos na rede de atenção à saúde (BRASIL, 2011).

Cuidados paliativos são, no mais das vezes, cuidados intensivos de conforto e gestão do fim da vida. Contudo, deverão ser iniciados logo no diagnóstico, intensificando-se na medida da necessidade. Consistem num direito do ser humano de ser apoiado e assistido no processo de doença até a fase final da vida. Os cuidados paliativos afirmam a vida e tratam a morte como um processo normal, e não implicam apressar ou adiar a morte. Nesse contexto, integram os aspectos psicossociais e espirituais nos cuidados ao paciente, oferecendo um sistema de apoio e ajuda aos mesmos para que vivam tão ativamente quanto possível até a morte. Além disso, disponibilizam um sistema de apoio para auxiliar o paciente e sua família a lidar com a situação durante a doença e no processo de luto; exigem uma abordagem em equipe, dando continuidade à atenção até o desfecho da necessidade de sua permanência na dinâmica familiar. Finalmente, afirmam que são aplicáveis desde o estágio inicial da doença, passando pelas modificações e terapias que prolongam a vida, até o processo de luto encerrado (WHO, 2002).

Os cuidados paliativos tiveram seu marco histórico na década de 1960, especialmente a partir da fundação do Saint Christopher's Hospice, em Londres, pela $\operatorname{Dr}^{\mathrm{a}}$ Cicely Saunders. Recentemente, têm conquistado reconhecimento científico no moderno movimento hospice - que defende o cuidar de um ser humano que está morrendo e de sua família, com compaixão e escuta empática. Propugna a filosofia do cuidado mais humanitário aos indivíduos com suas doenças crônicas e as que ameacem a vida. Inicialmente conhecidos como assistência hospice, o termo cuidados paliativos foi sendo absorvido pela comunidade científica, dentro de uma perspectiva que inclui, além dos cuidados administrados, o ensino e a pesquisa (FLORIANI; SCHRAMM, 2007; SILVA; HORTALE, 2006).

Os artigos constitucionais que versam sobre a saúde (art. 196 a 200) são regulamentados pelas Leis Orgânicas da Saúde (LOS) - Lei 8.080, de 19/09/1990, e Lei 8.142, de 28/12/1990. Segundo a Lei 8.080/90, o SUS pode ser compreendido como o conjunto de ações e serviços de saúde prestados por órgãos e instituições públicas federais, estaduais e municipais, da administração direta e indireta e das fundações mantidas pelo Poder Público. A Lei 8.142/90 garante a participação da comunidade na gestão do sistema de saúde e versa, ainda, sobre as transferências intergovernamentais de recursos financeiros na área da saúde. O SUS prevê um sistema com princípios doutrinários e organizativos e é constantemente atualizado por meio de leis, normas e regulamentações que vão sendo promulgadas em função de adequações a novas necessidades da população.

Para tanto, os princípios doutrinários do SUS (universalidade, equidade e integralidade) dizem respeito às ideias filosóficas que permeiam a implementação do sistema e consolidam o conceito ampliado de saúde e o princípio do direito à saúde. Os princípios organizativos (regionalização, hierarquização, participação do cidadão e descentralização) orientam a forma como o sistema deve funcionar, tendo como eixo norteador os princípios doutrinários (RONCALLI, 2003; FALEIROS ET AL., 2006).

Os princípios doutrinários do SUS são aqueles que traduzem a ideologia do novo modelo de saúde proposto para o País, reiterando o seu alcance e a forma de garantir o direito à saúde de todos os cidadãos. Entre eles, a universalidade - que encara a saúde como um direito ampliado de cidadania para todos - representa o fim do modelo excludente anterior, em que somente 
contribuintes da previdência social tinham direito a uma assistência à saúde mais qualificada. De uma maneira geral, portanto, considerando a política assistencial proposta pelo SUS, garantir acesso universal e equitativo é apenas uma das estratégias redistributivas contempladas em uma política de proteção e inclusão social. As políticas de saúde que têm como base a equidade exigem que seja definido esse conceito. No caso brasileiro, equidade foi definida a partir da igualdade no acesso, como nos deixa claro o artigo 196, da Constituição da República Federativa do Brasil, de 1988, que fala em acesso universal e igualitário. A política de saúde brasileira foi pensada num modelo prevencionista e aglutinador de ações onde pudesse atender todos os cidadãos indistintamente e que também corrigisse o modelo excludente do passado, tornando-se uma das políticas sociais do Estado brasileiro mais inclusivas e igualitárias. Finalmente, guardando coerência com a mudança de modelo proposta na doutrina, a integralidade compõe o seu ideário, considerando a visão não mais parcializada e fragmentada do cuidado, seja no nível das ações e dos serviços, seja na perspectiva do usuário que necessita de atenção preventiva e curativa (CONASS, 2007).

\section{Integralidade}

A integralidade é um dos princípios doutrinários do SUS e deve ser entendida como um princípio relativo à prática de saúde, aplicada tanto ao ato médico individual quanto à abordagem dos coletivos humanos, observando que cada pessoa é um todo indivisível e integrante de uma comunidade; as ações de promoção, proteção e recuperação da saúde formam, também, um todo indivisível e não podem ser compartimentalizadas; e as unidades prestadoras de serviço, com seus diversos graus de complexidade, formam, também, um todo indivisível, configurando um sistema capaz de prestar assistência integral. Em síntese,
O homem é um ser integral, bio-psico-social, e deverá ser atendido com essa visão integral por um sistema de saúde também integral, voltado a promover, proteger e recuperar sua saúde. (RONCALLI, 2003, P. 11).

O conceito de cuidados paliativos, na perspectiva da integralidade, diz respeito às várias dimensões e especificidades no tratamento do paciente e de sua família, como se pode observar no que foi estabelecido pela Organização Mundial de Saúde:

Cuidados Paliativos é uma abordagem que aprimora a qualidade de vida dos pacientes e famílias que enfrentam problemas associados com doenças ameaçadoras da vida, através da prevenção e alívio do sofrimento, por meio de identificação precoce, avaliação correta e tratamento da dor e outros problemas de ordem física, psicossocial e espiritual. (WHO, 2002, P. 84).

As diversas dimensões expressas a seguir estão diretamente relacionadas ao cuidado integral do paciente por parte dos profissionais e das equipes cuidadoras (WHO, 2002; BRASIL, 2005A; SILVA; HORTALE, 2006). São elas: (1) física - cuidados ativos e integrais centrados no indivíduo e em sua família, respeitando seus desejos e vontades; (2) psicológica avaliação do impacto da doença ameaçadora da vida no indivíduo e em seus familiares; (3) social - avaliação das necessidades sociais do indivíduo e de sua família através de uma abordagem individualizada e integrada; apresentação e discussão com a comunidade sobre a importância dos cuidados paliativos; inclusão da proposta de cuidados paliativos na formulação de políticas sociais e de saúde pública; (4) espiritual - respeito e consideração aos dogmas e/ou crenças religiosas, reconhecendo-as; suporte espiritual e religioso para indivíduos e familiares; (5) cultural atendimento às necessidades culturais dos indivíduos e familiares, por parte dos serviços de cuidados paliativos, que deve refletir 
a diversidade cultural da comunidade a qual serve; instituição de programas educacionais para os profissionais que atendam a essa diversidade cultural; (6) estrutural - formação de equipes interdisciplinares, incorporando voluntários; incorporação de atividades de melhoria da qualidade dos serviços; de pesquisa clínica e de processos gerenciais.

Para uma assistência integral em cuidados paliativos, todas essas dimensões deverão estar em perfeita sintonia com os cuidados integrais que a PNPCC-RAS propugna. Além de assinalar que fazem parte do cuidado integral a prevenção, a detecção precoce, o diagnóstico, o tratamento oportuno e seguro dos pacientes diagnosticados com câncer e lesões precursoras, a política recomenda, ainda, que os princípios e diretrizes de organização das ações e dos serviços baseados em evidências científicas devem ser considerados.

\section{Equidade}

Equidade é assegurar ações e serviços de todos os níveis de acordo com a complexidade que cada caso requeira, considerando que todo cidadão é igual perante o SUS e será atendido conforme suas necessidades. A equidade, definida como uma forma superior de justiça, vai considerar que toda a iniquidade se caracteriza por uma diferença sistemática que afeta a vida dos indivíduos de forma injusta, desnecessária e totalmente evitável, pois estabelece diferenças que são moralmente inaceitáveis. A promoção da equidade em saúde é fundamentalmente um compromisso ético e uma posição política que orienta as ações para assegurar o direito à saúde (BRASIL, 1990; BUSS; PELEGRINNI FILHO, 2007; NEGRI- FILHO, 2008).

No texto da PNPCC-RAS, a equidade é investida como responsabilidade dos entes federativos, especialmente no caso da regulação entre os componentes da rede de atenção à saúde, com definição de fluxos para fins de controle do acesso e da garantia do atendimento, promovendo a otimização de recursos segundo a complexidade e a densidade tecnológica necessárias à atenção da pessoa com câncer. $O$ texto salienta, também, a implantação do acolhimento e a humanização da atenção, com base em um modelo centrado no usuário e em suas necessidades de saúde, respeitando as diversidades étnico-raciais, culturais, sociais, territoriais e religiosas.

Em agosto de 2013, o Inca passa a integrar o sistema de regulação de vagas (Sisreg), em consonância com a política de prevenção e controle do câncer, que também é responsável pela organização do acesso às ações e aos serviços especializados referentes ao cuidado das pessoas com câncer. O sistema deve atuar de forma integrada, com garantia da transparência e da equidade no acesso, independentemente da natureza jurídica dos estabelecimentos de saúde, instituindo mecanismos de regulação do acesso para qualificar a demanda e a assistência prestada ao paciente com câncer (BRASIL, 2013).

No caso da PNPCC-RAS, além da otimização do sistema de regulação, uma das formas mais evidentes de vinculação ao princípio da equidade é a garantia da atenção em cuidados paliativos o mais próximo possível do domicílio do usuário, observando os critérios de atendimento domiciliar garantido pelo SUS, conforme a Lei 10.424, de 15/04/2002. Com atendimento multiprofissional e oferta de cuidado compatível com cada nível de atenção, a evolução da doença e a prioridade na realização de tratamento dos casos raros ou muito raros devem atender à exigência do alto nível de especialização e maior aporte tecnológico. Em síntese, na conceituação oficial, equidade significa assegurar ações e serviços de todos os níveis de acordo com a complexidade exigida pelos cidadãos em suas necessidades distintas, no âmago da política nacional (BRASIL, 1990).

\section{Universalidade}

O princípio da universalidade da atenção incorpora o direito à assistência como 
constructo da cidadania e aponta para um modelo que pressupõe uma lógica de seguridade social baseada nos moldes dos Estados de Bem-Estar Social. Os avanços do SUS são significativos, mas persistem problemas a serem enfrentados para consolidá-lo como um sistema público universal e equânime, para garantir uma saúde global para seus cidadãos.

No caso dos cuidados paliativos, o princípio da universalidade, ao pressupor a abrangência de todos os cidadãos na política distributiva do SUS, não os distingue desse tipo de cuidado. Significa dizer que todos, indistintamente, independentemente de sua classe econômica e social, são acolhidos pela política nacional de prevenção e controle do câncer. É sabido que os cidadãos de maior poder aquisitivo, embora possuam, na sua grande maioria, planos privados de saúde, têm muita dificuldade de obter destes a cobertura necessária no caso de câncer em sua vertente terminal, na qual a vigência de cuidados paliativos é imperiosa, seja pela complexidade da atenção, com utilização de tecnologia, muitas vezes, de alta densidade, $\mathrm{e}$ um alto grau de abordagem interdisciplinar; seja pela especificidade dos casos, implicando uma linha de cuidado com alto grau de referenciamento; ou seja, ainda, pela longevidade do cuidado, com grande imprevisibilidade temporal do desfecho da doença. Assim, os cuidados paliativos reiteram a doutrina universal do sistema de saúde, quando atendem a maioria da população brasileira, em todas as faixas de renda, reafirmando a filosofia generosa e universal do sistema ao garantir os cuidados de saúde a todos os seus cidadãos. Os cuidados paliativos, por respeitarem todas as dimensões do ser humano e se preocuparem com sua dignidade e autonomia, atendem aos preceitos éticos e morais defendidos pela Constituição brasileira. Por certo, não se observa essa mesma preocupação nos planos privados de saúde, apesar de, no senso comum, ostentarem um ideário de saúde pretensamente inovador e resolutivo.
A rigor, os cidadãos portadores de casos graves de câncer, dependentes de tratamento qualificado, como são os cuidados paliativos, acabam sendo 'devolvidos' pelos planos privados ao sistema público, aumentando, com isso, a demanda por essa modalidade.

\section{Discussão e considerações}

A prática em cuidados paliativos tende a crescer. Estima-se que, no País, a cada ano, cerca de 650 mil pessoas necessitem recorrer a essa modalidade de atenção, e $80 \%$ desse número corresponde a pacientes com câncer (FUNDAÇÃO DO CÂNCER, 2014). Essa realidade vai exigir uma resposta mais qualificada da política de saúde brasileira, necessitando estar ancorada numa perspectiva de apoio global aos múltiplos problemas dos pacientes que se encontram na fase mais avançada da doença e no final da vida.

As preocupações com relação aos cuidados paliativos, no Brasil, são relativamente novas. Elas ganham densidade quando são incorporadas na Política Nacional de Atenção Oncológica, em 2005, que foi reeditada em 2013, dentro do Plano Estratégico de Controle e Prevenção das DCNT. Entretanto, ainda não temos uma resposta satisfatória frente à crescente demanda dessa modalidade. A Organização Mundial de Saúde, em 1990, passou a se preocupar com a padronização dessa demanda, formulando, com isso, uma conceituação universal sobre cuidados paliativos, tentando, na época, organizar uma atenção do cuidado mais integral e humanizada àquelas pessoas acometidas por doenças terminais. Em face de novas necessidades e do crescente aumento de doenças e agravos à saúde da população com características crônicas e evolutivas, esse conceito foi redimensionado em 2002, contemplando, assim, uma maior abordagem aos pacientes e seus familiares em várias dimensões da vida humana. 
No Brasil, apesar das iniciativas de incorporar esse conceito e essa metodologia mesmo fundamentadas em bases legais, não existe ainda na rede de assistência à saúde uma política que dê conta de uma implementação e organização dos cuidados paliativos nos três níveis de complexidade do sistema de saúde. Para corroborar esse dado, a Associação Latino-Americana de Cuidados Paliativos (ALACP), em lançamento oficial de um atlas (PASTRANA ET AL., 2012) que versa sobre a qualidade e implantação dos cuidados paliativos na América do Sul, aponta que o Brasil ainda carece de maiores discussões, formação de profissionais, fomento de uma política específica e, logicamente, de uma organização onde seja preconizada a assistência em cuidados paliativos em todas as suas abrangências, ou seja, na atenção básica, na média e na alta complexidade.

Para uma assistência efetiva em cuidados paliativos, deve-se estabelecer interlocuções das várias áreas de atenção à saúde. $\mathrm{Na}$ atenção básica, eixo estruturante do sistema de saúde, onde a formação continuada dos agentes ganha importância fundamental, ainda não se observa contemplada no rol de suas ações uma formação específica em cuidados paliativos. Apesar de, recentemente, iniciativas, como o Programa Melhor em Casa, tentarem dar uma assistência em cuidados paliativos domiciliares, ainda não demonstram impacto na rede de atenção, conforme observado na revisão bibliográfica e documental efetuada, onde não há registros indicativos de cobertura dessa modalidade.

No nível da assistência de média complexidade, tem-se um verdadeiro espaço vazio com relação à atenção paliativa. Segundo o documento da ALACP, não temos, no Brasil, sequer um serviço completo de cuidados paliativos na média complexidade. Isso mostra um enorme descompasso dos serviços, uma vez que pacientes internados nesse nível, com suas doenças crônicas evolutivas e terminais, são lançados à própria sorte, recebendo, muitas vezes, um tratamento indigno na fase de maior complexidade da doença. Exemplo desse fato é a acolhida, já na fase terminal da doença, em unidades de terapia intensiva, em que o paciente, além de não ter sido cuidado previamente, sofre um tratamento invasivo e não resolutivo. $\mathrm{O}$ que caracteriza, segundo Gaudencio e Messeder (2011), o dilema, na grande maioria dos casos, entre o que deve ser feito e o que não deve ser feito por se tratar de paciente terminal.

O nível terciário, formado pelos Centros e Unidades de Alta Complexidade em Oncologia, cujos critérios são estabelecidos pela Portaria $N^{\circ} 741 / 2005$, não atende aos pré-requisitos recomendados. A Portaria é clara ao formular que, para o cadastramento e funcionamento dessas unidades, é necessário garantir: a atenção em cuidados paliativos, no que tange à assistência ambulatorial multiprofissional; as internações por intercorrências; as internações de longa permanência; e o fornecimento de medicação ou procedimentos de controle da dor.

É evidente a fragmentação do cuidado e a não efetividade no planejamento, uma vez que, na maioria dos casos, as medidas são isoladas e não implicam um tratamento integral aos pacientes encaminhados à rede de atenção oncológica. $\mathrm{O}$ 'Relatório de auditoria da política nacional de atenção oncológica', realizado pelo Tribunal de Contas da União (TC $\mathrm{n}^{\circ}$ 031.944/2010-8), corrobora essa situação. Buscando responder se a estrutura da rede de atenção oncológica vem possibilitando aos pacientes com câncer acesso tempestivo e equitativo a diagnóstico e a tratamento, assim se manifesta:

As análises desenvolvidas com base na documentação levantada sobre o tema e a partir das entrevistas realizadas durante os trabaIhos de campo evidenciaram que a rede de atenção oncológica não está suficientemente estruturada para possibilitar aos pacientes de câncer acesso tempestivo e equitativo ao diagnóstico e ao tratamento de câncer. (BRASIL, 2010, P. 20). 
Exceção a essa regra, no setor público, é o tratamento do paciente com câncer avançado oferecido pela unidade de cuidados paliativos do Instituto Nacional de Câncer José Alencar Gomes da Silva, que funciona como um hospice - considerado unidade de atenção completa em cuidados paliativos, sendo uma das poucas iniciativas do Brasil.

Uma efetiva mudança do modelo desfocado e fragmentado de assistência em cuidados paliativos encontrados no Brasil depende de uma atenção integral, digna e resolutiva, onde os princípios implementados pelo SUS sejam respeitados e realizados de forma a garantir o modelo integral, universal, igualitário e equânime ao portador de câncer avançado.

Para tanto, a capacitação dos profissionais da rede de assistência à saúde em cuidados paliativos se faz imperiosa, tanto no nível de formação acadêmica, nas modalidades de graduação, especialização, e até mesmo na formação de pesquisadores, com o intuito de fortalecer as bases científicas para o melhor aprimoramento do cuidado.

Estabelecer relações, parcerias, convênios com outros países, para o aprimoramento científico e tecnológico, principalmente com aqueles que já avançaram nessa discussão, como Argentina e Chile, na América do Sul, e Portugal, na União Europeia, que tem legislação e implementação dos cuidados paliativos bem consolidados, é outra tarefa urgente a ser assumida pelo setor público.

Outro aspecto relevante é estimular e desenvolver uma forte discussão com o controle social em saúde, instituído pela Lei ${ }^{\circ}$ $8142 / 1990$, como as comunidades, os pacientes, familiares e outras representações sociais, em observância às diretrizes constitucionais que determinam a participação do cidadão no sistema de saúde brasileiro.

No tocante à intersetorialidade das instâncias políticas, as áreas do transporte, na garantia da acessibilidade; da seguridade social, garantindo direitos sociais; da justiça, garantindo o acesso e a equidade, são imperiosas na formulação das políticas de saúde na perspectiva de sistema, como está proposto. Na perspectiva intrassetorial do sistema de saúde, cabe estabelecer relações estreitas com as diversas especialidades médicas, como geriatria, neurologia, psiquiatria, pediatria e as diversas categorias multiprofissionais que atuam em oncologia, além das áreas de informação epidemiológica e gestão, entre outras.

À instrumentalização de uma política nacional de cuidados paliativos que contemple $o$ cidadão no direito de ter direito, no direito de ter saúde e no direito de ter uma assistência no final da vida, corresponde, consequentemente, o direito de ter uma morte digna ou, melhor dizendo, um fim mais digno para a vida.

\section{Referências}

BRASIL. Ministério da Saúde. Portaria n 2.439/GM, de 8 de dezembro de 2005. Institui a Política Nacional de Atenção Oncológica: Promoção, Prevenção, Diagnóstico, Tratamento, Reabilitação e Cuidados Paliativos, a ser implantada em todas as unidades federadas, respeitadas as competências das três esferas de gestão. Diário Oficial [da] União, Brasília, DF, n. 76, 9 dez. 2005a. Seção 1, p. 80-81.

\footnotetext{
Ministério da Saúde. Portaria no 741/GM, de 19 de dezembro de 2005, com Anexos: Definem as Unidades de Assistência de Alta Complexidade em Oncologia, os Centros de Assistência de Alta Complexidade em Oncologia (CACON) e os Centros de Referência de Alta Complexidade em Oncologia e suas aptidões e qualidades e inclui outras providências. Diário Oficial [da] União, Brasília, DF, dez. 23 2005b. Seção 1, p. 113.
} 
Ministério da Saúde. Portaria nº 874/ GM, de 16 de Maio de 2013. Institui a Política Nacional para a Prevenção e Controle do Câncer na Rede de Atenção à Saúde das Pessoas com Doenças Crônicas no âmbito do Sistema Único de Saúde (SUS). Diário Oficial [da] União, Brasília, DF, 17 maio 2013, Seção 1, p.129-132.

Ministério da Saúde. Secretaria de Vigilância em Saúde. Departamento de Análise de Situação de Saúde. Plano de ações estratégicas para o enfrentamento das doenças crônicas não transmissíveis (DCNT) no Brasil 2011-2022. Brasília, DF: Ministério da Saúde, 2011.

Ministério da Saúde. Secretaria Nacional de Assistência à Saúde. ABC do SUS: doutrinas e princípios. Brasília, DF: Ministério da Saúde, 1990.

Tribunal de Contas da União. Relatório de Auditoria Operacional na Política Nacional de Atenção Oncológica. Brasília, DF, 2010.

BUSS, P. M.; PELLEGRINI FILHO A. A Saúde e seus Determinantes Sociais. Physis, Rio de Janeiro, v.17, n.1, 2007, p. 77-93.

CONSELHO NACIONAL DE SECRETÁRIOS DE SAÚDE (CONASS). Coleção Progestores Sistema Único de Saúde - Para entender a gestão do SUS, Brasília, DF: Ministério da Saúde, 2007.

FALEIROS, V. P. et al. A construção do SUS: história da Reforma Sanitária e do processo participativo. Brasília, DF: Ministério da Saúde, 2006.

FUNDAÇÃO DO CÂNCER. Cuidados Paliativos. Disponível em: <http:// www.cancer.org.br/projeto/93/ cuidados-paliativos>. Acesso em: 15 ago. 2014.

\section{FUNDAÇÃO OSWALDO CRUZ (FIOCRUZ). A saúde} no Brasil em 2030: diretrizes para a prospecção estratégica do sistema de saúde brasileiro. Rio de Janeiro: Fiocruz: Ipea: Ministério da Saúde: Secretaria de Assuntos Estratégicos da Presidência da República, 2012.

FLORIANI, C. A; SCHRAMM, F. R. Desafios morais e operacionais da inclusão dos cuidados paliativos na rede de atenção básica. Cad. Saúde Pública, Rio de Janeiro, v. 23, n. 9, set. 2007, p. 2072-2080.

GAUDENCIO, D.; MESSEDER, O. Dilemas sobre o fim-da-vida: informações sobre a prática médica nas UTIs. Ciênc. saúde colet., Rio de Janeiro, v. 16, supl.1, p. 813-820, 2011

HENNEMANN-KRAUSE, L. Ainda que não se possa curar, sempre é possível cuidar. Cuidados Paliativos. Revista Hospital Universitário Pedro Ernesto, Rio de Janeiro, v. 11, abr./jun. 2012, p. 13-17.

\section{INSTITUTO BRASILEIRO DE GEOGRAFIA e}

ESTATÍSTICA (IBGE). Indicadores Sociodemográficos e de Saúde no Brasil [internet]. 2009. Disponível em: $<$ http://www.ibge.gov.br/home/estatistica/populacao/ indic_sociosaude/2009/>. Acesso em: 20 jan. 2015.

\section{INSTITUTO NACIONAL DE CÂNCER JOSÉ} ALENCAR GOMES DA SILVA (INCA). Estimativa 2014: incidência de câncer no Brasil. Coordenação Geral de Ações Estratégicas, Coordenação de Prevenção e Vigilância. Rio de Janeiro: INCA, 2014.

\section{INSTITUTO NACIONAL DE CÂNCER JOSÉ DE} ALENCAR GOMES DA SILVA (INCA). Coordenação Geral de Ações Estratégicas, Coordenação de Prevenção e Vigilância Diretriz para a vigilância do câncer relacionado ao trabalho. Rio de Janeiro: INCA, 2012.

LIMA-COSTA, M. F. Epidemiologia do envelhecimento no Brasil. In: ROUQUAYROL, Z; ALMEIDA FILHO; N. (Org.). Epidemiologia \&t Saúde. 6. ed. Rio de Janeiro: MEDSI; 2003.

NEGRI-FILHO. A. A human rights approach to quality of life and health: applications to public health programming. International Journal, v. 10, n. 1, 2008.

PASTRANA.T et al. Atlas de Cuidados Paliativos en Latinoamérica. Houston: International Association for Hospice and Palliative Care. 1. ed. 2012.

RONCALLI, A. G. O desenvolvimento das políticas públicas de saúde no Brasil e a construção do Sistema Único de Saúde. In: PEREIRA, C. P. (Org.). Odontologia 
em Saúde Coletiva: planejando ações e promovendo saúde. Porto Alegre: ARTMED, 2003.

SILVA, R. C. F; HORTALE, V. A. Cuidados paliativos oncológicos: elementos para o debate de diretrizes nesta área. Rio de Janeiro, Cad. Saúde Pública, Rio de Janeiro, v. 22, n. 10, p. 2055-2066, out. 2006.
WORLD HEALTH ORGANIZATION (WHO). National cancer control programmes: policies and managerial guidelines. 2. ed. Genebra: OMS, 2002.

Recebido para publicação em outubro de 2014

Versão final em janeiro de 2015

Conflito de interesse: inexistente

Suporte financeiro: não houve 